\title{
On varieties closed under the construction of power algebras
}

\section{A. Shafaat}

It is shown that if a variety $V$ of (universal) algebras, defined by a set $\Sigma$ of identities, is closed under the construction of power algebras then $V$ can be defined by the subset $\Sigma^{\prime}$ of $\Sigma$ consisting of those identities $v=w$ of $\Sigma$ such that every variable in $v=w$ occurs exactly once on both sides.

\section{Introduction}

Let $A$ be an $\Omega$-algebra [2], where $\Omega$ is a set of non-nullary "operators". By the power algebra of $A$ is meant the $\Omega$ algebra $P(A)$ consisting of all subsets of $A$ and with $\omega \in \Omega$ defined by

$$
\left(s_{1}, \ldots, s_{n}\right) \omega=\left\{\left(s_{1}, \ldots, s_{n}\right) \omega ;\left(s_{1}, \ldots, s_{n}\right) \in s_{1} \times \ldots \times s_{n}\right\}
$$

for all $S_{1}, \ldots, S_{n} \subseteq A$, where $n$ is the "arity" of $\omega$.

We shall say that a class $K$ of $\Omega$-algebras is P-closed or closed under $P$ or closed under the construction of power algebras if $A \in K$ implies $P(A) \in K$.

Let $X$ be a fixed infinite set of "variables" and let $W_{X}$ be the $\Omega$-word algebra [2] on $X$. A word $w \in W_{X}$ will be called linear if every $x \in X$ occurs at most once in $v$; an identity [2] $v=w$ will be called linear if both $v, w$ are linear. The relevance of linearity to the study of $P$-closed classes arises from the fact that for a linear

Received 3 May 1974. 
$w\left(x_{1}, \ldots, x_{n}\right) \in W_{X}$ and for $s_{1}, \ldots, s_{n} \in P(A)$ we can write

$$
w\left(s_{1}, \ldots, s_{n}\right)=\left\{w\left(s_{1}, \ldots, s_{n}\right) ; s_{1} \in s_{1}, \ldots, s_{n} \in s_{n}\right\} .
$$

This is not possible in general. For example, if $\Omega$ consists of a binary operation + and $w=x_{1}+x_{1}$ then $s_{1}+s_{-1}$ is not $\left\{s_{1}+s_{1} ; s_{1} \in s_{1}\right\}$ unless $S_{1}$ has only one element.

By a regular identity is meant an identity in which every variable that occurs on one side also occurs on the other. It is easily verified, and known [4], that a variety $V$ of $\Omega$-algebras is $P$-closed if it is defined by a set of linear, regular identities. For the converse of this fact the following two theorems are known (see [1], [3] and [4]).

THEOREM 1. If $V$ is P-closed and is defined by a single identity $v=w$ then $v=w$ is either regular, linear or of the form $v=v$.

THEOREM 2. Every P-closed variety is definable by a set of regular, linear identities.

The purpose ${ }^{1}$ of this note is to give the following synthesis of the above two theorems.

THEOREM 3. If $V$ is a P-closed variety defined by a set $\Sigma$ of identities then $V$ can be defined by the (possibly empty) set of regular, inear identities in $\Sigma$.

Theorem 2 follows directly from Theorem 3. Theorem 1 can also be easily deduced from our theorem as follows: If $\Sigma$ consists of a single identity $v=w$ and defines a $P$-closed variety then, by Theorem $3, \Sigma$ is equivalent to the subset $\Sigma^{\prime}$ of regular, linear identities of $\Sigma$. Then $\Sigma^{\prime}$ is empty or else $\Sigma^{\prime}=\Sigma$. In the latter case $v=w$ must be regular, linear while in the former $v=w$ must define the variety of all $\Omega$-algebras and hence must be of the form $v=v$.

\section{Proof of Theorem 3}

We begin with a few definitions which our proof requires. For every $v \in W_{X}$ and $x \in X$ we write $d_{x}(v)$ for the number of occurrences of $x$ 1 I would like to thank Mr S. Whitney, who discovered Theorem 2 independently of [1], for pointing out to me that Gautam's Theorem I does not follow 'directly' from Theorem 2 and for other useful remarks. 
in $v$. We refer to $d_{x}(v)$ as the degree of $x$ in $v$. By the degree of $v$ we mean the largest integer, to be denoted by $d(v)$, in the (finite) set $\left\{d_{x}(v) ; x \in X\right\}$. For every finite set $V$ of words we define $d(V)$ to be the largest of the integers $d(v), v \in V$, if $V$ is non-empty and $d(V)=0$ if $V$ is empty. For every finite set $\Sigma$ of identities we define $d(\Sigma)=d(V)$, where $V=\{v, w ; v=w \in \Sigma\}$. We write $d(\{v=w\})$ simply as $d(v=w)$. We shall refer to $d(V), d(\Sigma)$, and so on, as the degree of $V, \Sigma$, and so on.

An identity will.be called balanced if $d_{x}(v)=d_{x}(w)$ for all $x \in X$; that is, if every variable occurs the same number of times in $v$ as in $w$. It is immediate that a balanced identity is regular. Also, an identity is regular and linear if and only if it is balanced and of degree 1 .

Lemma 1 and Lemma 2 below cover a good part of our proof of Theorem 3. The first of these two lemmas hardly needs any proof.

LEMMA 1.

(1.1) An identity of the form $v=v$ is balanced.

(1.2) If $v=w$ is balanced then so is $w=v$ and $d(v=w)=d(w=v)$.

(1.3) If $u=v$ and $v=w$ are balanced then so is $u=w$ and $d(u=v)=d(v=w)=d(u=w)$.

(1.4) If $v\left(x_{1}, \ldots, x_{n}\right)=w\left(x_{1}, \ldots, x_{n}\right)$ and $u=u^{\prime}$ are batanced then so is $v\left(u, x_{2}, \ldots, x_{n}\right)=w\left(u, x_{2}, \ldots, x_{n}\right)$ and the degree of $v\left(u, x_{2}, \ldots, x_{n}\right)=w\left(u^{\prime}, x_{2}, \ldots, x_{n}\right)$ is not smaller than that of $v=w$.

The above lemma shows that rules of deduction of identities from identities preserve the property of being balanced. Also, the rules of deduction do not cause the degree to decrease. This makes the following lemma intuitively clear.

LEMMA 2. Let $v=w$ be deducible from a finite set $\Sigma$ of balanced identities but not from any other subset of $\Sigma$. Then $v=w$ is balanced and $d(\Sigma) \leq d(v=w)$. 
Proof. If $\Sigma$ is empty then $v=w$ must be of the form $v=v$ and the required result follows from Lemma $l(1.1)$ and $d(\Sigma)=0$. Assume therefore that $\Sigma$ is non-empty. Then $v, w$ must be distinct; for, otherwise $v=w$ is deducible from the empty subset of $\Sigma$ which is distinct from $\Sigma$, contradicting our assumption.

Let $v_{1}=w_{1}, \ldots, v_{n-1}=w_{n-1}, v=w$ be a proof of $v=w$ (from $\Sigma$ ) of shortest possible length $n$. If $n=1$ then $v=w$ must belong to $\Sigma$ and hence is deducible from the subset $\{v=w\}$ of $\Sigma$; we must therefore have $\Sigma=\{v=w\}$ which gives $d(\Sigma) \leq d(v=w)$.

Let $n>1$ (so that $v=w$ is not a member of $\Sigma$ ) and using induction on $n$ assume that the lemma is true for all $\Sigma^{\prime}$ and $v^{\prime}=w^{\prime}$ such that $v^{\prime}=w^{\prime}$ can be deduced from $\Sigma^{\prime}$ in less than $n$ steps.

Since $v, w$ are distinct, therefore $v=w$ is not a result of reflexivity. Nor is $v=w$ a member of $\Sigma$. Hence $v=w$ is obtained from one or two of the identities $v_{1}=w_{1}, \ldots, v_{n-1}=w_{n-1}$ by an application of one of the rules:

(I) symmetry;

(2) transitivity;

(3) substitution of a variable as in Lemma 1 (1.4).

That $v=w$ is balanced is now clear from Lemma 1 . We show that $d(\Sigma) \leq d(v=w)$.

For every $i, 1 \leq i \leq n-1$, let $\Sigma_{i}$ denote a minimal subset of $\Sigma$ from which $v_{i}=w_{i}$ can be deduced. Let $v=w$ be obtained from $v_{i}=w_{i}$ by an application of (1). Then $v=w$ is clearly deducible from $\Sigma_{i}$. By our assumption on $\Sigma$ this implies $\Sigma_{i}=\Sigma$ and the induction hypothesis gives $d(\Sigma)=d\left(\Sigma_{i}\right) \leq d\left(v_{i}=w_{i}\right) \leq d(v=w)$, where the last inequality follows from (1.2) of Lemma 1 .

Now let $v=w$ be obtained from $v_{i}=w_{i}, v_{j}=w_{j}$ by an application of (2) or (3). Then, as above, we must have $\Sigma=\Sigma_{i} \cup \Sigma_{j}$. Hence, by the induction hypothesis and (1.3) or (1.4) of Lemma 1, we get 
$d(\Sigma)=\max \left(d\left(\Sigma_{i}\right), d\left(\Sigma_{j}\right)\right) \leq \max \left(d\left(v_{i}=w_{i}\right), d\left(v_{j}=w_{j}\right)\right) \leq d(v=w)$.

This proves the lemma.

Since every identity deducible from a set $\Sigma$ of identities is deducible from a finite subset of $\Sigma$ we have

COROLLARY 1. Every identity deducible from a set of balanced identities is itself balanced.

The above corollary and Theorem 2 give

COROLLARY 2. Every identity of a P-closed variety is balanced.

To prove Theorem 3 let now $V$ be a $P$-closed variety defined by a set of identities $\Sigma$. Let $\Sigma^{\prime}$ be the subset of $\Sigma$ consisting of regular, linear identities. By Theorem 2, $V$ can be defined by the set $\Sigma^{\prime \prime}$ of all regular, linear identities of $V$. Since $\Sigma^{\prime} \subseteq \Sigma^{\prime \prime}$ we need only show that $\Sigma^{\prime \prime}$ is deducible from $\Sigma^{\prime}$. Let $v=w$ be an identity of $\Sigma^{\prime \prime}$ and let $\Delta$ be a minimal subset of $\Sigma$ from which $v=w$ is deducible; $\Delta$ exists because $\Sigma$ and $\Sigma^{\prime \prime}$ are equivalent. We have to show that $\Delta \subseteq \Sigma^{\prime}$. If $\Delta$ is empty then nothing remains to be proved. Otherwise, by Lemma 2 , $1 \leq d(\Delta) \leq d(v=w)=1$. Hence $d(\Delta)=1$, which implies that $\Delta$ consists of balanced identities of degree 1 . This proves $\Delta \subseteq \Sigma^{\prime}$ and hence the theorem.

A straightforward modification of the above proof will give

THEOREM 4. Let $\Gamma_{n}$ be the set of all balanced $\Omega$-identities of degree less than a given positive integer $n$. Let $\Sigma$ be a set of s-identities which is equivalent to a subset of $\Gamma_{n}$. Then $\Sigma$ is equivalent to $\Sigma \cap \Gamma_{n}$.

\section{References}

[1] M.N. Bleicher, Hans Schneider and R.L. Wilson, "Permanence of identities on algebras", Algebra Universalis 3 (1973), 72-93.

[2] P.M. Cohn, Universal algebra (Harper and Row, New York, Evanston, London, 1965). 
[3] N.D. Gautam, "The validity of equations of complex algebras", Arch. Math. Logik Grondlagenforsch. 3 (1957), 117-124.

[4] Stephen Whitney, "Model classes of linear theories", submitted.

Département de Mafhématique,

Université Laval,

Cité Universitaire,

Québec,

Canada. 